\title{
Noise through Quantum Pumps
}

\author{
M. L. Polianski ${ }^{a}$, M. G. Vavilov ${ }^{b, a}$, and P. W. Brouwer ${ }^{a}$ \\ ${ }^{a}$ Laboratory of Atomic and Solid State Physics, Cornell University, Ithaca, NY 14853 \\ ${ }^{b}$ Theoretical Physics Institute, University of Minnesota, Minneapolis, MN 55455
}

(October 28, 2018)

\begin{abstract}
We study the current noise through an unbiased quantum electron pump and its mesoscopic fluctuations for arbitrary temperatures and beyond the bilinear response. In the bilinear regime, we find the full distributions of the noise power and the current-to-noise ratio for a chaotic quantum dots with single-channel and many-channel ballistic point contacts. For a dot with many-channel point contacts we also calculate the ensemble-averaged noise at arbitrary temperature and pumping strength. In the limit of strong pumping, a new temperature scale appears that corresponds to the broadening of the electron distribution function in the dot as a result of the time-dependent perturbations.
\end{abstract}

PACS numbers: 73.23.-b, 72.10.Bg, 72.70.+m

\section{INTRODUCTION}

A periodic perturbation of a confined electron system may produce a direct current. The initial theoretical proposals [1.2] and experimental realizations [3 5] for electron pumps were for a pump where the spectrum is gapped and the charge pumped in one cycle quantized and not subject to fluctuations. Recently, attention has shifted to pumps that are well connected to electron reservoirs, and, hence, do not have a gapped excitation spectrum [6] 9]. If the pump relies on a time-dependent perturbation that mainly affects the quantummechanical phases of the electrons, and not their classical trajectories, it is referred to as a "quantum electron pump". Such a quantum pump was fabricated by Switkes et al. [10].

The current that is pumped through a quantum electron pump is subject to mesoscopic fluctuations and to quantum or thermal fluctuations (noise). Mesoscopic fluctuations of the current refer to the fact that the magnitude and direction of the time-averaged current vary from sample to sample. For a quantum pump built from a chaotic quantum dot, as is the case in the experiment of Ref. [10], the mesoscopic current fluctuations were investigated for various regimes of temperature, pumping amplitude and dot conductances [8,9, 11, 14]. On the other hand, noise - quantum and thermal fluctuations of the current - is a property of the current pumped through a particular realization of an electron pump. Noise in an electron pump is best described by the fluctuations of the charge pumped through the system in a certain number of pumping cycles. The statistics of such charge fluctuations was studied in Refs. 15 22] for temperatures and pumping frequencies much smaller than the inverse dwell time (escape rate) of electrons in the quantum dot.

In this paper, we consider the mesoscopic fluctuations of the noise. We do not impose any restrictions on the relative magnitudes of temperature $T$, pumping frequency $\omega$, escape rate $\gamma$ and pumping amplitude. This is important, as in an experimental realization of a quantum pump, $T$ and $\gamma$ are usually comparable, while both are much larger than $\omega$. Previous works by Andreev and Kamenev [15] and Levitov [16] addressed the full counting statistics, but at temperatures $k T \ll \hbar \omega$ only. The mean square charge fluctuations for $\hbar \omega, k T \ll \gamma$ (but including the case $\hbar \omega \approx k T$ ) were considered very recently by Moskalets and Büttiker [22].

Denoting the quantummechanical average with a bar $\overline{-}$, the quantum and thermal fluctuations of the pumped charge are described by

$$
S=\frac{1}{\tau_{0}}\left[\bar{Q}^{2}-(\bar{Q})^{2}\right] .
$$

Here $\tau_{0}$ is the observation time and $Q$ is the total charge pumped through the dot in the time $\tau_{0}$. The noise in an electron pump can be divided onto a Nyquist-Johnson component $S^{N}$ and pumping component $S^{P}$. The former is the thermal equilibrium noise due to the thermal fluctuations of electrons in the leads, and depends on the electron temperature $T$ in the leads and the timeaveraged conductance $\bar{G}$ of the quantum dot through the fluctuation-dissipation theorem,

$$
S^{N}=2 k T \bar{G} .
$$

The pumping contribution $S^{P}$, in contrast, is a true nonequilibrium noise due to the perturbation of electrons inside the dot. As we shall discuss in Sec. III, this contribution to the noise can be seen as arising due to the heating of electrons inside the dot as a result of the timedependent perturbation.

In the adiabatic regime $\hbar \omega \ll \gamma$ one needs to vary at least two system parameters periodically in order to generate a direct current. Current noise, however, is already generated if only one parameter is varied. The problem of current noise (and full counting statistics) for a single time-dependent scatterer with frequency $\hbar \omega \ll \gamma$ was addressed by Levitov and coworkers 19.20. Our results can be used to compute the mesoscopic fluctuations of the current in that case. We find that the main effect 
of the second time-dependent parameter in a true electron pump is to reduce the mesoscopic fluctuations of the noise.

The paper is organized as follows: A formal expression for the noise in terms of the time-dependent scattering matrix of the $\operatorname{dot} \mathcal{S}$ is derived in Sec. II. Section III considers the mesoscopic fluctuations of the noise through a chaotic quantum dot with two (or more) time-dependent parameters. For an adiabatic quantum pump at temperature $k T \ll \gamma$ we consider the full distribution of the mesoscopic fluctuations of the noise in Sec. III A. We focus on the cases of a quantum dot with singlechannel and many-channel point contacts. For a dot with many-channel point contacts, the sample-to-sample fluctuations of the noise are much smaller than the average. In Sec. III B we then present the ensemble-averaged noise for a dot with many-channel point contacts at arbitrary temperature and pumping strength. We conclude in Sec. IV. Finally, in Appendix we give some details of the calculations of the averages over the ensemble of chaotic quantum dots.

\section{GENERAL FORMALISM}

We consider fluctuations of charge transmitted the sample during the observation time $\tau_{0}$. The sample is connected to electron reservoirs through two point contacts with $N_{L}$ and $N_{R}$ open channels, respectively, see Fig. 1. It is subject to a periodic perturbation at frequency $\omega / 2 \pi$; The perturbation is described by specifying the time-dependence of parameters $X_{1}(t), X_{2}(t)$, $\ldots, X_{n}(t)$ characterizing Hamiltonian of the sample. The electrons in the two reservoirs are held at the same chemical potential $\mu$ and temperature $T$ at all times during the pumping cycle.

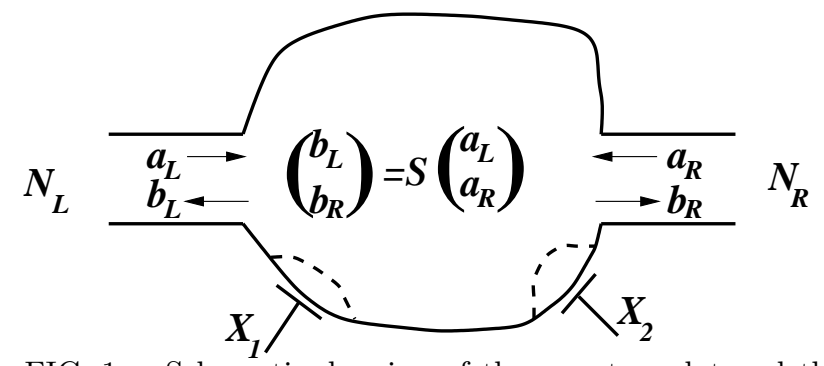

FIG. 1. Schematic drawing of the quantum dot and the leads. There are $N_{L}\left(N_{R}\right)$ propagating modes in the left (right) contact. The shape of the dot is controlled by the voltages of two shape-defining gates. The vectors $\mathbf{a}_{L, R}$ and $\mathbf{b}_{L, R}$ of annihilation operators for the incoming and outgoing states, respectively, in the left $(\mathrm{L})$ and right $(\mathrm{R})$ leads are related by the scattering matrix $\mathcal{S}$.

We calculate the noise of the quantum pump using the scattering formalism of Büttiker [23. In Büttiker's original application there are no time-dependent pertur- bations, so that the system is described by a scattering matrix that depends on energy, but not on time. On the other hand, as long as pumping frequencies and temperatures much smaller than the escape rate $\gamma$ are considered, the system with a time-dependent perturbation can be described using a scattering matrix that depends on time, but not on energy 15,16,19 22. When $k T, \hbar \omega$ and $\gamma$ are all comparable, one needs to use a scattering matrix $\mathcal{S}$ that depends on both time and energy, or, equivalently, that depends on two times or two energies. Here we shall make use of a formulation with a scattering matrix $\mathcal{S}\left(t, t^{\prime}\right)$ that depends on two times. This formulation was used to calculate the the time-averaged conductance and pumped current in the Ref. 13]. The formalism is equivalent to the two-energy scattering formalism developed by Büttiker and coworkers for time-dependent transport through mesoscopic structures 2427.

The scattering matrix $\mathcal{S}\left(t, t^{\prime}\right)$ relates the annihilation [creation] operators $\mathbf{a}_{\alpha}(t)\left[\mathbf{a}^{\dagger}(t)\right]$ and $\mathbf{b}_{\alpha}(t)\left[\mathbf{b}_{\alpha}^{\dagger}(t)\right]$ of incoming states and outgoing states in channel $\alpha=$ $1, \ldots, 2 N$ of the leads (the index $\alpha$ includes the spin degree of freedom),

$$
\begin{aligned}
& \mathbf{b}_{\alpha}(t)=\int_{-\infty}^{+\infty} \mathcal{S}_{\alpha \beta}\left(t, t^{\prime}\right) \mathbf{a}_{\beta}\left(t^{\prime}\right) d t^{\prime}, \\
& \mathbf{b}_{\alpha}^{\dagger}(t)=\int_{-\infty}^{+\infty} \mathbf{a}_{\beta}^{\dagger}\left(t^{\prime}\right)\left(\mathcal{S}^{\dagger}\left(t^{\prime}, t\right)\right)_{\beta \alpha} d t^{\prime} .
\end{aligned}
$$

Here the indices $\alpha$ and $\beta$ label the propagating channels in the point contact contacting the dot to the left and right reservoirs for $\alpha, \beta=1, \ldots, 2 N_{L}$ and $\alpha, \beta=$ $2 N_{L}+1, \ldots, 2 N$, respectively. The $2 N \times 2 N$ matrices $\mathcal{S}^{\dagger}$ and $\mathcal{S}$ are related as

$$
\left(\mathcal{S}^{\dagger}\left(t^{\prime}, t\right)\right)_{\alpha \beta}=\mathcal{S}_{\beta \alpha}^{*}\left(t, t^{\prime}\right)
$$

Causality requires that $\mathcal{S}\left(t, t^{\prime}\right)=0$ if $t<t^{\prime}$. We restrict our attention to the case where spin rotation invariance is preserved, and, hence, $\mathcal{S}$ is proportional to the $2 \times 2$ unit matrix in spin grading.

The expression for the current $\mathbf{I}_{L}$ in the left lead is

$$
\mathbf{I}_{L}(t)=e \sum_{\alpha=1}^{2 N_{L}}\left[\mathbf{a}_{\alpha}^{\dagger}(t) \mathbf{a}_{\alpha}(t)-\mathbf{b}_{\alpha}^{\dagger}(t) \mathbf{b}_{\alpha}(t)\right]
$$

A similar expression holds for the current $\mathbf{I}_{R}$ in the right lead. Since we are only interested in the charge pumped through the dot in a long time interval and the charge on the dot is conserved after each cycle, we replace the expression for the current operator by a suitable combination of $\mathbf{I}_{L}$ and $\mathbf{I}_{R}$,

$$
\begin{aligned}
\mathbf{I}(t) & =\frac{N_{R}}{N} \mathbf{I}_{L}(t)-\frac{N_{L}}{N} \mathbf{I}_{R}(t) \\
& =e \sum_{\alpha, \beta=1}^{2 N}\left(\mathbf{a}_{\alpha}^{\dagger}(t) \Lambda_{\alpha \beta} \mathbf{a}_{\beta}(t)-\mathbf{b}_{\alpha}^{\dagger}(t) \Lambda_{\alpha \beta} \mathbf{b}_{\beta}(t)\right) .
\end{aligned}
$$


Here $\Lambda$ is a diagonal matrix with elements

$$
\Lambda_{\alpha \alpha}= \begin{cases}N_{R} / N & \alpha=1, \ldots, 2 N_{L}, \\ -N_{L} / N & \alpha=2 N_{L}+1, \ldots, 2 N .\end{cases}
$$

In terms of the current operator $\mathbf{I}$, the time-averaged pumped current $I$ reads

$$
I=\frac{1}{\tau_{0}} \int_{0}^{\tau_{0}} d t \overline{\mathbf{I}}(t)
$$

where the observation time $\tau_{0}$ is an integer number of pumping cycles. The noise $S$ is defined as [cf. Eq. (1)]

$$
S=\frac{1}{\tau_{0}} \int_{0}^{\tau_{0}} d t d t^{\prime}\left(\overline{\mathbf{I}(\mathbf{t}) \mathbf{I}\left(\mathbf{t}^{\prime}\right)}-\overline{\mathbf{I}}(t) \overline{\mathbf{I}}\left(t^{\prime}\right)\right) .
$$

In the leads, the electron distribution function is given by the Fourier transform $f(t)$ of the Fermi function,

$$
\begin{aligned}
& \overline{\mathbf{a}_{\beta}^{\dagger}\left(t^{\prime}\right) \mathbf{a}_{\alpha}(t)}=\delta_{\alpha \beta} f\left(t^{\prime}-t\right), \\
& \overline{\mathbf{a}_{\beta}\left(t^{\prime}\right) \mathbf{a}_{\alpha}^{\dagger}(t)}=\delta_{\alpha \beta} \tilde{f}\left(t-t^{\prime}\right),
\end{aligned}
$$

where we defined

$$
\tilde{f}(t)=\delta(t)-f(t)
$$

Using Eq. (3) to eliminate the operators $\mathbf{b}(t)$ and $\mathbf{b}^{\dagger}(t)$ from Eq. (6a) and Eq. (9) to compute the quantummechanical expectation values, we find the average current

$$
\begin{aligned}
I= & \frac{1}{\tau_{0}} e \int_{0}^{\tau_{0}} d t \int d t_{1} d t_{2} f\left(t_{1}-t_{2}\right) \\
& \times \operatorname{Tr}\left[\mathcal{S}^{\dagger}\left(t_{1}, t\right) \Lambda \mathcal{S}\left(t, t_{2}\right)-\delta\left(t-t_{1}\right) \Lambda \delta\left(t-t_{2}\right)\right],
\end{aligned}
$$

and the noise

$$
S=S^{N}+S^{P}
$$

where the Nyquist-Johnson and pumping contributions $S^{N}$ and $S^{P}$ read

$$
\begin{aligned}
S^{N}= & \frac{2 e^{2}}{\tau_{0}} \int_{0}^{\tau_{0}} d t d t^{\prime} \int d t_{1} d t_{2} f\left(t_{1}-t^{\prime}\right) \tilde{f}\left(t^{\prime}-t_{2}\right) \operatorname{Tr}\left[\delta\left(t-t_{1}\right) \Lambda^{2} \delta\left(t-t_{2}\right)-\mathcal{S}^{\dagger}\left(t_{1}, t\right) \Lambda \mathcal{S}\left(t, t_{2}\right) \Lambda\right], \\
S^{P}= & \frac{e^{2}}{\tau_{0}} \int_{0}^{\tau_{0}} d t d t^{\prime} \int d t_{1} d t_{2} d t_{1}^{\prime} d t_{2}^{\prime} f\left(t_{1}-t_{2}^{\prime}\right) \tilde{f}\left(t_{1}^{\prime}-t_{2}\right) \\
& \times \operatorname{Tr}\left[\mathcal{S}^{\dagger}\left(t_{1}, t\right) \Lambda \mathcal{S}\left(t, t_{2}\right) \mathcal{S}^{\dagger}\left(t_{1}^{\prime}, t^{\prime}\right) \Lambda \mathcal{S}\left(t^{\prime}, t_{2}^{\prime}\right)-\delta\left(t-t_{1}\right) \delta\left(t^{\prime}-t_{1}^{\prime}\right) \Lambda^{2} \delta\left(t-t_{2}\right) \delta\left(t^{\prime}-t_{2}^{\prime}\right)\right] .
\end{aligned}
$$

(These equations can also be derived using the Keldysh formalism, see Ref. [28].)

For adiabatic pumping, $\hbar \omega \ll \gamma$, Equation (11) is equivalent to the time-averaged current of Refs. [8] and [9]. The Nyquist-Johnson contribution to the noise is related to the time-averaged conductance $\bar{G}$ of the system at temperature $T$, see Refs. 29, 30 and Eq. (2) above.

\section{A. Bilinear adiabatic pumping}

Of particular interest is the case when the perturbation is slow compared to the (elastic) escape rate $\gamma$ of the electrons from the sample into the reservoirs. This is the regime of the adiabatic quantum pump of Ref. [10]. In this approximation it is advantageous to use an analog of the Wigner transform for the matrix $\mathcal{S}\left(t, t^{\prime}\right)$ :

$$
\mathcal{S}(\varepsilon, t)=\int_{-\infty}^{+\infty} d t^{\prime} e^{-i \varepsilon\left(t-t^{\prime}\right)} \mathcal{S}\left(t, t^{\prime}\right)
$$

Up to corrections of order $\hbar \omega / \gamma$, the matrix $\mathcal{S}(\varepsilon, t)$ is equal to the "instantaneous" scattering matrix $\mathcal{S}_{X}(\varepsilon)$, which is obtained by "freezing" all parameters $X_{j}$ to their values at time $t$ 31, 32].

If, in addition to being adiabatic, the parameters $X_{j}$, $j=1, \ldots, n$, undergo only small excursions from their average value, which we set to zero, we may further expand in $X_{j}$. To find the noise it is sufficient to expand $\mathcal{S}$ up to the second order in $X$. Arranging the parameters $X_{j}$ in an $n$-component vector $\mathcal{X}=\left(X_{1}, \ldots, X_{n}\right)^{\mathrm{T}}$, we thus find

$$
\begin{aligned}
S^{P}= & \frac{e^{2}}{4 \tau_{0}} \int_{0}^{\tau_{0}} d t d t^{\prime} \int_{-\infty}^{+\infty} \frac{d \varepsilon}{2 \pi} \int_{-\infty}^{+\infty} \frac{d \varepsilon^{\prime}}{2 \pi} \cos \left[\left(\varepsilon-\varepsilon^{\prime}\right)\left(t-t^{\prime}\right)\right]\left[f(\varepsilon) \tilde{f}\left(\varepsilon^{\prime}\right)+f(\varepsilon) \tilde{f}\left(\varepsilon^{\prime}\right)\right] \\
& \times\left[\left(\mathcal{X}^{\mathrm{T}}(t)-\mathcal{X}^{\mathrm{T}}\left(t^{\prime}\right)\right) \mathcal{K}\left(\varepsilon, \varepsilon^{\prime}\right)\left(\mathcal{X}\left(t^{\prime}\right)-\mathcal{X}(t)\right)\right]
\end{aligned}
$$

where $\tilde{f}(\varepsilon)=1-f(\varepsilon)$ and the $n \times n$ matrix $\mathcal{K}$ reads

$$
\mathcal{K}_{i j}\left(\varepsilon, \varepsilon^{\prime}\right)=\operatorname{Tr}\left[\Lambda^{2} \mathcal{R}_{i}(\varepsilon) \mathcal{R}_{j}(\varepsilon)+\Lambda^{2} \mathcal{R}_{j}\left(\varepsilon^{\prime}\right) \mathcal{R}_{i}\left(\varepsilon^{\prime}\right)-2 \Lambda \mathcal{R}_{i}(\varepsilon) \Lambda \mathcal{R}_{j}\left(\varepsilon^{\prime}\right)\right], \quad \mathcal{R}_{j}(\varepsilon)=-i \frac{\partial \mathcal{S}_{X}(\varepsilon)}{\partial X_{j}} \mathcal{S}_{X}^{\dagger}(\varepsilon) .
$$


In the bilinear regime and at zero temperature, the time-averaged current $I$ and the noise $S^{P}$ are sufficient to define the total counting statistics of the pump [16]: To lowest order in the excursions of the parameters $X_{j}$, the pumping cycles are statistically independent. A pumping cycle is characterized by quantummechanical probabilities $P_{R}$ and $P_{L}$ that an electron is pumped from left to right or from right to left, respectively. Both $P_{R}$ and $P_{L}$ are small, of order $X^{2}$, and one has $I=(e \omega / 2 \pi)\left(P_{L}-P_{R}\right), S^{P}=\left(e^{2} \omega / 2 \pi\right)\left(P_{L}+P_{R}\right)$.

For a typical quantum dot with capacitance $C$ and mean level spacing $\Delta$, the charging energy $e^{2} / 2 C \gg \Delta$. The derivatives in Eq. (16b) should be taken at a constant value of the chemical potential $\mu$, which, in the Hartree approximation, is equal to sum of the electron's kinetic energy and the electrostatic potential. In the absence of electron-electron interactions all derivatives are taken at constant value of the kinetic energy. As we prefer to take derivatives at constant kinetic energy (i.e., at constant $\varepsilon$ ) in both cases, we substitute the parametric derivatives as 24, 33.

$$
\left.\left.\frac{\partial}{\partial X}\right|_{\mu} \rightarrow \frac{\partial}{\partial X}\right|_{\varepsilon}-\left(\frac{\frac{1}{2} \operatorname{Tr} \mathcal{R}_{i}}{\frac{\pi C}{e^{2}}-\frac{i}{2} \operatorname{Tr} \frac{\partial \mathcal{S}_{X}}{\partial \varepsilon} \mathcal{S}_{X}^{\dagger}}\right) \frac{\partial}{\partial \varepsilon} .
$$

Equation (17) differs from a similar expression in Ref. [8] by factor of $1 / 2$ in front of the traces because of the double size of matrix $\mathcal{S}$ as a result of the inclusion of spin.

\section{APPLICATION TO CHAOTIC QUANTUM DOTS}

We now consider the mesoscopic fluctuations of the pumping noise $S^{P}$ for the case of a chaotic quantum dot. The quantum dot is characterized by a mean level spacing $\Delta$, escape rate $\gamma=N \Delta / 2 \pi$, Thouless energy $E_{\mathrm{Th}}=\hbar / \tau_{\mathrm{erg}} \gg \Delta$, and capacitance $C$, with charging energy $e^{2} / 2 C \gg \Delta$. It is coupled to two electron reservoirs via ballistic point contacts with $N_{L}$ and $N_{R}$ channels each, see Fig. 1 .

The dot is driven by periodically varying parameter(s) $X_{i}, i=1, \ldots, n$ with frequency $\omega$, cf. Fig. 1. In the experiment of Ref. [10], the parameters $X_{i}$ correspond to the voltages on external gates that control the dot shape. The precise relation between the parameters $X$ used in the theory and in the experiment is not known a priori, but can be established using independent measurements of, e.g., the derivative of the conductance 33] or the rate of change of the position of Coulomb blockade peaks when the point contacts between the dot and the reservoirs are pinched off [13]. Following Refs. [8, 11, 13], we will assume that the different parameters $X_{i}$ correspond to different perturbations of which the matrix elements between states (of the closed dot) within a Thouless energy from the Fermi level $\varepsilon=0$ are Gaussian and independently distributed. We choose the scale for the parameters $X_{i}$ such that the mean square derivative $\left\langle\left(\partial \varepsilon_{\mu} / \partial X_{i}\right)\left(\partial \varepsilon_{\mu} / \partial X_{j}\right)\right\rangle=\delta_{i j} \Delta^{2} / \pi^{2}$, where $\Delta$ is the mean level spacing and $\varepsilon_{\mu}$ is an energy level in the closed dot.

The transmitted charge $Q$ is measured during a time $\tau_{0}$, which we will assume to be a large number of pumping cycles. This requirement of large observations is discussed in detail in Ref. [20]. For short observation times boundary effects related to switching processes in the system need to be taken into account.

\section{A. Weak adiabatic low temperature pumping}

If not only the frequency $\hbar \omega$ is much smaller than the escape rate $\gamma$, but also $k T \ll \gamma$, the scattering matrix $S(\varepsilon, t)$ in Eq. (16) can be taken at the Fermi level $\varepsilon=0$, and we find the simple result

$$
\begin{aligned}
S^{P} & =\frac{e^{2}}{\tau_{0}} \int_{0}^{\tau_{0}} d t d t^{\prime} f\left(t-t^{\prime}\right) \tilde{f}\left(t^{\prime}-t\right) \\
& \times \frac{1}{2}\left[\left(\mathcal{X}(t)^{\mathrm{T}}-\mathcal{X}\left(t^{\prime}\right)^{\mathrm{T}}\right) \mathcal{K}\left(\mathcal{X}\left(t^{\prime}\right)-\mathcal{X}(t)\right)\right], \\
\mathcal{K}_{i j} & =\operatorname{Tr}\left(\Lambda^{2} \mathcal{R}_{i} \mathcal{R}_{j}+\Lambda^{2} \mathcal{R}_{j} \mathcal{R}_{i}-2 \Lambda \mathcal{R}_{i} \Lambda \mathcal{R}_{j}\right) .
\end{aligned}
$$

We now consider the case of two time-dependent parameters, $\mathcal{X}(t)=\left(X_{1}(t), X_{2}(t)\right)^{\mathrm{T}}$ :

$$
X_{1}(t)=X_{1} \cos (\omega t), X_{2}(t)=X_{2} \cos (\omega t+\phi),
$$

in more detail. For this case, Eq. (18a) is factorized into a factor $F\left(T, \omega, \tau_{0}\right)$ that depends on the relevant time and energy scales, and a sample specific contribution,

$$
\begin{aligned}
S^{P}= & e^{2} F\left(T, \omega, \tau_{0}\right)\left(\mathcal{K}_{11} X_{1}^{2}+\mathcal{K}_{22} X_{2}^{2}\right. \\
& \left.+2 \cos \phi \mathcal{K}_{12} X_{1} X_{2}\right) .
\end{aligned}
$$

To find the integral $F\left(T, \omega, \tau_{0}\right)$ for the physically relevant limit of long observation times $\tau_{0} \gg 1 / T$, we note that in this limit the distribution function $f(t)$ is given by Fourier transform of the equilibrium Fermi distribution function,

$$
f(t)=\int \frac{d \varepsilon}{2 \pi \hbar} \frac{e^{i \varepsilon t / \hbar}}{e^{\varepsilon / k T}+1}=\frac{i k T}{2 \hbar \sinh (\pi k T t / \hbar)} .
$$

Substitution into Eq. (18a) then yields

$$
F=\frac{\omega}{2 \pi}\left(\operatorname{coth} \frac{\hbar \omega}{2 k T}-\frac{2 k T}{\hbar \omega}\right) .
$$

In the opposite limit $\tau_{0} \ll \hbar / k T, F\left(T, \omega, \tau_{0}\right)$ can be found using the procedure proposed by Ivanov and Levitov [20]. In a finite observation time $\tau_{0}$, energy levels are resolved up to $\hbar / \tau_{0}$, so that we must set 


$$
f(t)=\frac{i}{2 \tau_{0}} \frac{1}{\sin \left[\pi(t+i 0) / \tau_{0}\right]} .
$$

Then, following Ref. 20], we change the time variable $t \in\left[0, \tau_{0}\right]$ to $z=e^{2 \pi i t / \tau_{0}}$, and perform contour integration in the circle $|z|=1$ in complex plane. The result coincides with the low-temperature limit of Eq. (22) above.

The physical meaning of the function $F\left(T, \omega, \tau_{0}\right)$ with $\tau_{0} \gg 1 / T$ becomes clear once it is written in energy representation,

$$
\begin{aligned}
F= & \int \frac{d \varepsilon}{2 \pi \hbar}\left[\frac{1}{2} f\left(\varepsilon+\frac{1}{2} \hbar \omega\right) \tilde{f}\left(\varepsilon-\frac{1}{2} \hbar \omega\right)-f(\varepsilon) \tilde{f}(\varepsilon)\right. \\
& \left.+\frac{1}{2} f\left(\varepsilon-\frac{1}{2} \hbar \omega\right) \tilde{f}\left(\varepsilon+\frac{1}{2} \hbar \omega\right)\right] .
\end{aligned}
$$

Equation (24) measures the change in the number of equilibrium electron-hole pairs due to absorption and emission of the pumping field quantum $\hbar \omega$. At temperatures $k T \ll \hbar \omega$ the Fermi distribution is sharp, and $F=\omega / 2 \pi$. At high $k T \gg \hbar \omega$, the Fermi distribution is smooth on the scale $\sim \omega$, so that $F$ is small, $F \sim \hbar \omega^{2} / k T$.

Fluctuations of the noise are described by the second factor in Eq. (20), which varies from sample to sample. To find the mesoscopic fluctuations of the noise, we use the joint distribution of the matrices $\mathcal{R}_{i}(i=1,2)$ derived in Ref. [34]. The resulting distribution of this mesoscopic contribution depends on two parameters,

$$
C_{l}=\frac{2}{N}\left(X_{1}^{2}+X_{2}^{2}\right), \quad C_{c}=\frac{4}{N} X_{1} X_{2} \sin \phi .
$$

Equality $C_{c}=C_{l}$ is achieved if $X_{1}=X_{2}$ and $\phi=\pi / 2$, corresponding to the circular contour in the $\left(X_{1}, X_{2}\right)$ plane. Below we consider the noise distribution for a quantum dot with single-channel point contacts $\left(N_{L}=\right.$ $N_{R}=1$ ), and for a dot with many-channel point contacts $\left(N_{L}, N_{R} \gg 1\right)$ separately.

\section{Two channel geometry, $N=2$}

For small $N$, the full distribution of the noise can be obtained using the method of Ref. [35] to numerically generate the matrices $\mathcal{R}_{1}$ and $\mathcal{R}_{2}$ according to the appropriate distribution, see Appendix for details. Figure 2 shows the distribution of the noise power $S^{P}$ and the current-to-noise ratio $I / S^{P}$ for a quantum dot with single-channel leads $(N=2)$ for the case $C_{l}=C_{c}$. The distributions are shown with and without time-reversal symmetry (TRS). For reference we have also included the distributions for the case $e^{2} / C \ll \Delta$ of weak electronelectron interactions inside the dot. The case $C_{c}<C_{l}$ (i.e., the dependence on the phase difference $\phi$ ) is illustrated in Fig. 3. The current-to-noise ratio shown in Figs. 2 and 3 accounts for the pumping noise $S^{P}$ only. The Nyquist-Johnson noise $S^{N}$ presents a different noise source, which will dominate over the pumping noise if $k T \gtrsim \hbar \omega \sqrt{C_{l}}$.

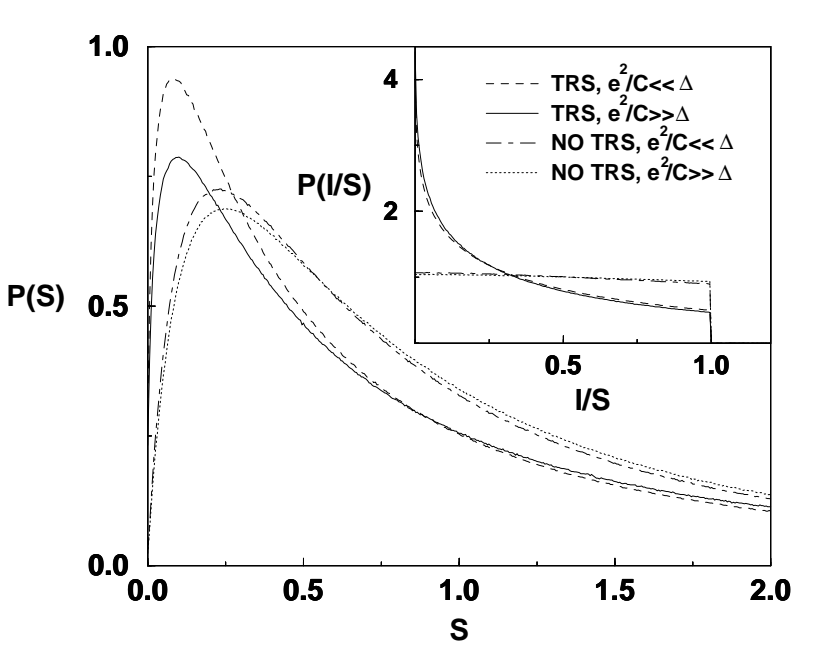

FIG. 2. Main panel: Distribution of pumping noise $S^{P}$ for a chaotic quantum dot with single-channel point contacts and two time-dependent parameters $X_{1}$ and $X_{2}$ given by Eq. (19) with $C_{l}=C_{c}$. [The parameters $C_{l}$ and $C_{c}$ are defined in Eq. (25).] The noise is measured in units of $e^{2} C_{l} F$. The plots are with and without time-reversal symmetry (TRS) and for $e^{2} / C \ll \Delta$ (weak electron-electron interactions inside the dot) or $e^{2} / C \gg \Delta$. Inset: Distribution of the current-to-noise ratio $I / S^{P}$, measured in units of $\omega /(2 \pi F e)$. There is no divergence at $I / S^{P} \rightarrow 0$.

We note that the distributions of $S^{P}$ and $I / S^{P}$ are highly non-Gaussian. In particular, the mean $\left\langle S^{P}\right\rangle$ of the noise distribution is dominated by the algebraic tail for large $S^{P}$, and is not representative of the distribution itself. [For example, in the absence of time-reversal symmetry and for $e^{2} / C \ll \Delta$, the mean $\left\langle S^{P}\right\rangle=8 / 3\left(e^{2} C_{l} F\right)$, while the most probable value is for $S^{P} \approx 0.5\left(e^{2} C_{l} F\right)$.] We also note that, while the phase difference $\phi$ affects the typical size of the time-averaged pumped current $I \propto e \omega C_{c}$ but not the form of the distribution, changing $\phi$ has a small effect on the average noise $\left\langle S^{P}\right\rangle$, but changes the shape of the noise distribution significantly, see Fig. 3. In particular, the probability to find small $S^{P}$ is significantly higher for $\phi$ close to zero than for $\phi \sim \pi / 2$. The reason for this difference is that the case $\phi \sim \pi / 2$ corresponds to noise generated by two independent sources, while $\phi$ close to zero corresponds to only a single noise source. Furthermore, the current-noise ratio $I / S^{P}$ has a maximum at $I / S^{P}=e$, as was predicted by Levitov [16]. For a quantum dot with single-channel point contacts, there is a finite probability density to achieve this optimum current-to-noise ratio, as is seen in the inset of Fig. 2. For point contacts with more than one channel, the probability density to attain the maximum value $I / S^{P}=e$ vanishes [16], see, e.g. , Fig. [4. 


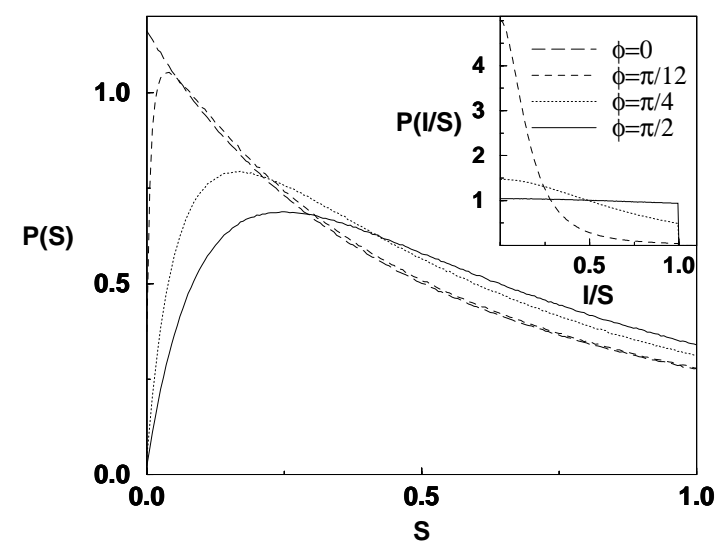

FIG. 3. Distribution of the pumping noise $S^{P}$ (main panel) and the current-to-noise ratio $I / S^{P}$ (inset) for a quantum dot with two single channel point contacts and broken time-reversal symmetry, for various values of $C_{c} / C_{l}$. The values of $C_{c} / C_{l}$ shown are $C_{c} / C_{l}=1, \sin (\pi / 4), \sin (\pi / 12)$, and 0 . If the variations of the parameters $X_{1}$ and $X_{2}$ have equal amplitudes, this corresponds to phase difference $\phi=\pi / 2, \pi / 4$, $\pi / 12$, and 0 , respectively. The noise $S^{P}$ is measured in units of $e^{2} C_{l} F$ and the current $I$ is measured in units of $e C_{l} \omega / 2 \pi$. For $C_{c}=0, P\left(I / S^{P}\right)$ is a delta function at $I / S^{P}=0$ (not shown).

\section{Multichannel limit, $N \gg 1$}

For large $N$, the ensemble average and variance of the noise can be expressed in terms of an integral over the unitary group and over the eigenvalues of the WignerSmith time-delay matrix $\mathcal{R}=-i \hbar(\partial S / \partial \varepsilon) S^{\dagger}[38]$. These integrals can be calculated using the method of Refs. [36,37, together with asymptotic expressions for the density and two-point correlations of the eigenvalues of the Wigner-Smith time-delay matrix, see Appendix for details.

The result is

$$
\begin{aligned}
\left\langle S^{P}\right\rangle= & 2 e^{2} F g\left(1+\frac{2}{N} \delta_{\beta, 1}\right) C_{l} \\
\operatorname{var} S^{P}= & \left(2 e^{2} F\right)^{2} \frac{g}{N}\left(3\left[1+\left(1+2 \delta_{\beta, 1}\right) \frac{2 g}{N}\right] C_{l}^{2}\right. \\
& \left.-\left[1+\left(1+4 \delta_{\beta, 1}\right) \frac{g}{N}\right] C_{c}^{2}\right)
\end{aligned}
$$

where we abbreviated $g=N_{L} N_{R} / N$. The factor $F$ depends on $\omega$ and $T$ and was defined in Eq. (22). Note that the fluctuations of $S^{P}$ are a factor $\sim 1 / N$ smaller than the average. Higher cumulants of the noise are even smaller, so that we conclude that, for $N \gg 1$, the noise distribution becomes sharply peaked at the average $\left\langle S^{P}\right\rangle$ and that the remaining sample-to-sample fluctuations are Gaussian.

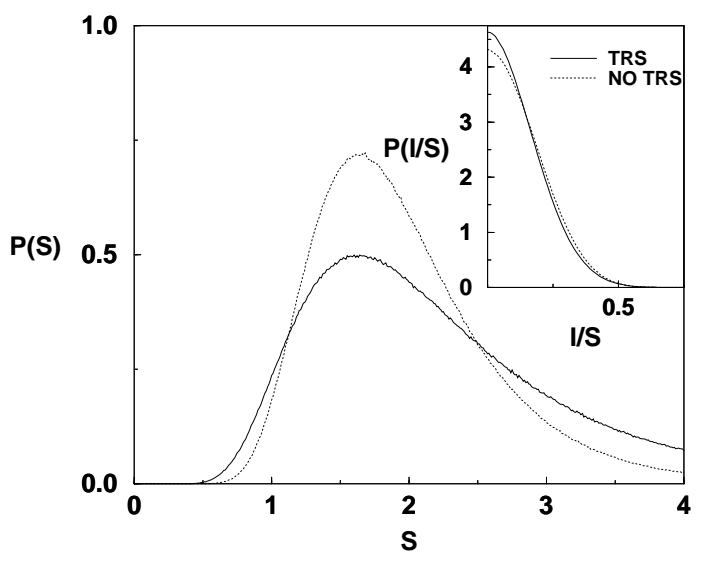

FIG. 4. The same as Fig. 2 for a quantum dot with $N_{L}=N_{R}=5$. Curves with weak and strong electron-electron interaction are indistinguishable.

In Fig. 1 we show the results of a numerical calculation of the distribution noise $S^{P}$ and the current-tonoise ratio $I / S^{P}$ for a dot with $N_{L}=N_{R}=5, N=10$. This value of $N$ can be seen as intermediate between the small- $N$ regime, where the distributions are strongly non-Gaussian and the large- $N$ regime where the distributions are Gaussian. We note that for $N=10$ the noise distribution still has pronounced tails for large $S^{P}$.

\section{B. Noise at arbitrary pumping for multichannel dots}

In this subsection we consider a general time dependence and amplitude of the parameters $X_{j}$. We limit ourselves to the calculation of the ensemble averaged noise, since the mesoscopic fluctuations of the noise are smaller than the average by a factor $1 / N$ if $N \gg 1$, see Eq. (26).

For a calculation of the ensemble average noise $\left\langle S^{P}\right\rangle$, we need to know the correlation functions of scattering matrix elements $\mathcal{S}_{\alpha \beta}\left(t, t^{\prime}\right)$ for an ensemble of chaotic quantum dots. The indices $\alpha$ and $\beta$ refer to the "orbital" channels as well as to the spin of the electrons. In order to discriminate between the orbital and spin degrees of freedom, we set $\alpha=(i, \sigma)$, where $\sigma= \pm 1$ refers to spin and $i=1, \ldots, N$ denotes the orbital channels, $i=1, \ldots, N_{L}$ for channels in the left point contact and $i=N_{L}+1, \ldots, N$ for channels in the right point contact. In this notation, the scattering matrix $\mathcal{S}_{\alpha \beta}=\mathcal{S}_{i j}^{\circ} \delta_{\sigma \sigma^{\prime}}$ is proportional to the $2 \times 2$ unit matrix in spin space. The correlator of the orbital part $S_{i j}^{\mathrm{o}}$ reads

$$
\left\langle\mathcal{S}_{i j}^{\mathrm{o}}\left(t, t^{\prime}\right) \mathcal{S}_{k l}^{\mathrm{o*}}\left(\tau, \tau^{\prime}\right)\right\rangle=\delta\left(t-t^{\prime}-\tau+\tau^{\prime}\right)\left[\delta_{i k} \delta_{j l} \mathcal{D}\left(\frac{t+\tau}{2}, t^{\prime}+\frac{\tau-t}{2}, \tau-t\right)+\delta_{i l} \delta_{j k} \mathcal{C}\left(\frac{t+\tau}{2}, t^{\prime}+\frac{\tau-t}{2}, \tau-t\right)\right],
$$


with $\mathcal{D}$ and $\mathcal{C}$ given by

$$
\begin{aligned}
& \mathcal{D}\left(t_{1}, t_{2}, \tau\right)=\Theta\left(t_{1}-t_{2}\right) \exp \left\{-\int_{t_{2}}^{t_{1}} \frac{\Delta d \xi}{2 \pi \hbar}\left[N_{d}+2\left(\mathcal{X}^{\mathrm{T}}(\xi-\tau / 2)-\mathcal{X}^{\mathrm{T}}(\xi+\tau / 2)\right)(\mathcal{X}(\xi-\tau / 2)-\mathcal{X}(\xi+\tau / 2))\right]\right\} \\
& \mathcal{C}\left(t_{1}, t_{2}, \tau\right)=\Theta\left(t_{1}-t_{2}\right) \exp \left\{-\int_{t_{2}-\tau / 2}^{t_{1}-\tau / 2} \frac{\Delta d \eta}{2 \pi \hbar}\left[N_{c}+2\left(\mathcal{X}^{\mathrm{T}}(\eta)-\mathcal{X}^{\mathrm{T}}\left(t_{1}+t_{2}-\eta\right)\right)\left(\mathcal{X}(\eta)-\mathcal{X}\left(t_{1}+t_{2}-\eta\right)\right)\right]\right\}
\end{aligned}
$$

Here $\Theta(z)=1$ if $z>0$ and 0 otherwise and we abbreviated

$$
N_{d}=N, \quad N_{c}=N+2 x^{2},
$$

where $x \propto\left(\Phi / \Phi_{0}\right)\left(\tau_{\operatorname{erg}} \Delta\right)^{-1 / 2}$ is a dimensionless parameter describing the magnetic flux penetrating the quantum dot and $\Phi_{0}$ the flux quantum [39]. The unitary ensemble, when time-reversal symmetry is fully broken, corresponds to the limit $x \rightarrow \infty$.

In the literature, two equivalent approaches have been taken to calculate correlators such as Eq. (27) above, the Hamiltonian and scattering approaches [39]. In the Hamiltonian approach the fundamental object is the random Hamiltonian of the closed chaotic quantum dot and the Green functions related to it. Once the scattering matrix $\mathcal{S}$ is expressed in terms of Green functions, the correlator (27) can be analyzed by standard diagrammatic techniques. The two terms $\mathcal{C}$ and $\mathcal{D}$ then appear as the cooperon and diffuson contributions. The fundamental object of the scattering approach is a statistical model for the scattering matrix $\mathcal{S}$ of an ensemble of dots. Equivalence of both methods, including the parametric and energy dependence of $\mathcal{S}$ is shown in Ref. [34]. A derivation of Eq. 227) using the scattering approach is given in Ref. 40]. We refer to Ref. 28] for a discussion based on the Hamiltonian approach.

Knowing the correlator (27), we can find the ensembleaveraged noise $\left\langle S^{P}\right\rangle$,

$$
\left\langle S^{P}\right\rangle=\frac{2 e^{2} g}{\tau_{0}} \int_{0}^{\tau_{0}} d t d t^{\prime} f\left(t-t^{\prime}\right) \tilde{f}\left(t^{\prime}-t\right)\left(\left[\int_{0}^{\infty} \mathcal{D}\left(\frac{t+t^{\prime}}{2}, \frac{t+t^{\prime}}{2}-\frac{\hbar \zeta}{\gamma}, t^{\prime}-t\right) d \zeta\right]^{2}-1\right),
$$

where $g=N_{L} N_{R} / N$ is the dot conductance, $\gamma=N \Delta / 2 \pi$ the escape rate, and we assumed $\tau_{0} \gg 1 / \omega, \hbar / \gamma$. The Cooperon contribution $\mathcal{C}$ of Eq. (27) does not contribute to $\left\langle S^{P}\right\rangle$ to leading order in $1 / N$; its contribution is a factor $1 / N$ smaller, see, e.g., Eq. (26a).

Equation (31) gives the ensemble-averaged noise for arbitrary $\omega, T$, and $\gamma$, and for arbitrary excursions of the parameters $X_{j}$. We now investigate Eq. (31) for the case that there are two time-dependent parameters $X_{1}$ and $X_{2}$ with time dependence given by Eq. (19). In order to distinguish regimes of "weak" (bilinear) and "strong" pumping, we introduce the energy scale [13,30]

$$
k T^{*}=\hbar \omega \sqrt{C_{l}} .
$$

The meaning of the energy scale $k T^{*}$ becomes clear when we view the pumping process as "diffusion in energy space": carriers absorb or emit energy quanta of size $\hbar \omega$ at a rate $X^{2} \Delta / \hbar$ [13]. Weak (bilinear) pumping corresponds to the case when the probability to absorb or emit one or more quanta is small, i.e., to the regime $X^{2} \Delta \ll \gamma$ or $k T^{*} \ll \hbar \omega$. For $k T^{*} \gg \hbar \omega$ many quanta are absorbed or emitted, so that the carriers in the dot shift their energies by an amount $\sim \hbar \omega \sqrt{X^{2} \Delta / \gamma} \sim T^{*}$. If $T^{*}$ exceeds the temperature $T$ of the electrons in the leads, the timedependent potentials in the dot lead to significant "heating" of the electrons inside the dot and $T^{*}$ can be viewed as an effective electron temperature inside the dot. The latter regime is referred to as "strong" pumping.

For weak pumping, $k T^{*} \ll \hbar \omega$, we can expand $\mathcal{D}$ to first order in $X^{2}$ and we recover the result Eq. (26a), now without a restriction on the temperature $T$. For strong pumping, $k T^{*} \gg \max \{\hbar \omega, k T\}$, a simple expression for the noise power can be obtained if pumping is adiabatic, $\hbar \omega \ll \gamma$. In that case, we note that $\mathcal{D}$ in Eq. (31) contains the fast decay $\sim \exp (-\zeta)$ and a slowly varying contribution from the time-dependence of the parameters $X_{j}$. Since the $X_{j}$ vary slowly on the time scale $\hbar / \gamma$, the integration over $\zeta$ can be done, and the result is

$$
\left\langle S^{P}\right\rangle=\frac{2 e^{2} g}{\tau_{0}} \int_{0}^{\tau_{0}} d t^{\prime} d t f\left(t^{\prime}-t\right) \tilde{f}\left(t-t^{\prime}\right)\left[\left(\frac{N}{N+2\left(\mathcal{X}^{\mathrm{T}}(t)-\mathcal{X}^{\mathrm{T}}\left(t^{\prime}\right)\right)\left(\mathcal{X}(t)-\mathcal{X}\left(t^{\prime}\right)\right)}\right)^{2}-1\right]
$$

In the limit of low temperatures, $k T \ll \hbar \omega$ (and, as before, assuming long observation times $\hbar / \tau_{0} \ll k T$, $\hbar \omega$ ), Eq. (33) yields

$$
\left\langle S^{P}\right\rangle=\frac{e^{2} g \omega}{\pi} \frac{\left[1+6 C_{l}\left(1+C_{l}\left(1-S^{2}\right)\right)\right] \mathbf{E}(k)-\left[1+2 C_{l}(1-S)\right] \mathbf{K}(k)}{\pi\left[1+2 C_{l}(1-S)\right] \sqrt{1+2 C_{l}(1+S)}},
$$


where $\mathbf{E}(k)$ and $\mathbf{K}(k)$ are full elliptic integrals of the 2nd and 3rd kind, respectively, and we abbreviated

$$
S=\sqrt{1-\left(\frac{C_{c}}{C_{l}}\right)^{2}}, \quad k^{2}=\frac{4 C_{l} S}{1+2 C_{l}(1+S)} .
$$

In the special case that the two time-dependent parameters $X_{1}(t)$ and $X_{2}(t)$ have equal amplitudes, $S=|\cos \phi|$. The dependence of the averaged noise in Eq. (34) on the ratio $C_{c} / C_{l}$ (i.e., on the phase difference $\phi$ ) is weak. For the case $C_{c}=0(\phi=0)$ we find the asymptotes

$$
\begin{aligned}
& \left\langle S^{P}\right\rangle=e^{2} g \frac{\omega}{\pi} C_{l}=e^{2} g \frac{\left(k T^{*}\right)^{2}}{\pi \hbar^{2} \omega} \text { if } k T^{*} \ll \hbar \omega \\
& \left\langle S^{P}\right\rangle=e^{2} g \frac{3 \omega}{\pi^{2}} \sqrt{\frac{C_{l}}{2}}=e^{2} g \frac{3}{\pi^{2} \hbar \sqrt{2}} k T^{*} \text { if } k T^{*} \gg \hbar \omega
\end{aligned}
$$

For the case $C_{c}=0$, but at arbitrary temperatures $k T$, Eq. (33) yields

$$
\left\langle S^{P}\right\rangle=e^{2} g \omega\left(\frac{2 k T}{\hbar \omega}\right)^{2} \int_{0}^{\infty} \frac{d \zeta}{\sinh ^{2}(2 \pi k T \zeta / \hbar \omega)}\left\{1-\frac{\hbar \omega}{\sqrt{(\hbar \omega)^{2}+4\left(k T^{*}\right)^{2} \sin ^{2} \zeta}}+\frac{2\left(k T^{*}\right)^{2} \hbar \omega \sin ^{2} \zeta}{\left[(\hbar \omega)^{2}+4\left(k T^{*}\right)^{2} \sin ^{2} \zeta\right]^{3 / 2}}\right\},
$$

The low temperature asymptotics can be easily obtained from this result, and reproduce the Eq. (36) for $k T^{*} \ll$ $\hbar \omega$ or $k T^{*} \gg \hbar \omega$. For intermediate values of $k T^{*} / \hbar \omega$ and $k T / \hbar \omega$, Eq. (37) is plotted in Fig. 5 . As long as both $\hbar \omega$ and $k T$ are much smaller than $k T^{*}$, the integral (37) is dominated by $\zeta \ll \hbar \omega / k T^{*} \ll 1$, so that the strong pumping asymptote of Eq. (36b) is reached, irrespective of $\omega$ or $T$.

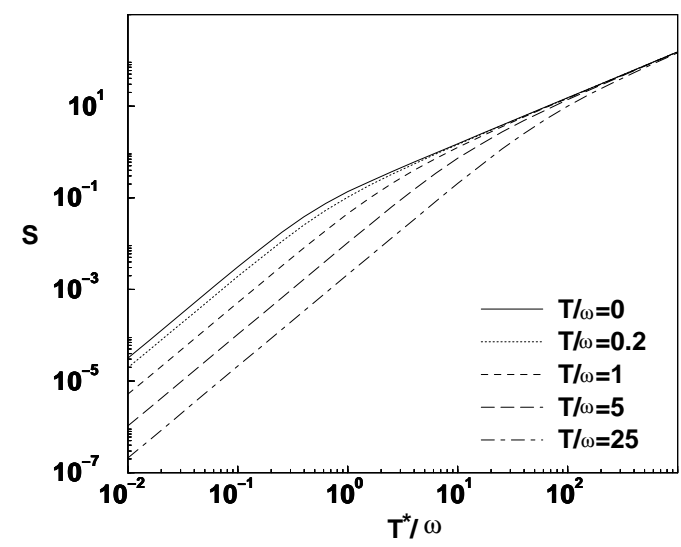

FIG. 5. Noise $\left\langle S^{P}\right\rangle$ in units of $e^{2} g \omega$, as a function of the dimensionless pumping strength $k T^{*} / \hbar \omega$ and for various choices $k T / \hbar \omega$.

An analytical expression for $\phi \neq 0$ can, in principle, be obtained from Eq. (33) as well. The qualitative behavior as a function of pumping strength and temperature is similar to that shown in Fig. 5 for the case $\phi=0$. The limit of strong pumping should converge to the limit Eq. (34) of $T=0$, similarly to the case $\phi=0$ studied above.

\section{CONCLUSION}

In this paper we calculated the current noise generated in a quantum pump using a scattering matrix formalism with a scattering matrix $\mathcal{S}\left(t, t^{\prime}\right)$ that depends on two times. With this formalism, we could consider arbitrary pumping frequency $\omega$, temperature $T$, escape rate $\gamma$, and pumping strength $X$. We then calculated the average and variance of the noise for an ensemble of quantum pumps consisting of a chaotic quantum dot.

One issue that has received considerable attention recently is the question whether one can build a noiseless quantum pump [41. While our results for the bilinear pumping regime show that there is a finite (mesoscopic) probability for zero noise, it is not possible to have no noise and a finite pumped current at the same time in the bilinear regime [16, 42], cf. Figs. 2 and 3. This is different beyond the bilinear regime, where a quantized, and, hence, noiseless pump has been proposed using a pumping contour that encircles a resonance in an almost closed quantum dot 43.

We note that both the equilibrium Nyquist-Johnson noise $S^{N}$ and the pumping noise $S^{P}$ depend on the available energy window. For pumping noise, that energy window is the heating temperature $T^{*}$, see Eq. (32); for Nyquist-Johnson noise it is the temperature $T$ of the electron reservoirs. The results (26) and (34)-(37) allow us to compare the Nyquist-Johnson and pumping contributions to the averaged noise. In the experimentally relevant case that $\hbar \omega \ll k T$, both noise contributions are proportional to the (dimensionless) dot conductance $g$, but the Nyquist-Johnson noise scales as $T$, while the pumping noise scales as $T^{*}$ if $T^{*} \gg T$ and as $T^{* 2} / T$ if $T^{*} \ll T$. The Nyquist-Johnson and pumping contribu- 
tions to the noise are comparable at $T \sim T^{*}$. An experiment cannot separate the two contributions to the noise, since it measures the total noise power. The pumping contribution to the noise is dominant as long as $T \ll T^{*}$.

\section{ACKNOWLEDGMENTS}

We would like to thank Igor Aleiner for discussions. There is some overlap between our results and a recent preprint of Moskalets and Büttiker (Ref. [22]), which appeared shortly before completion of our manuscript. This work was supported by the Cornell Center for Materials Research under NSF grant No. DMR-0079992, by the NSF under grants no. DMR-0086509 and DMR-0120702, and by the Sloan and Packard foundations.

\section{APPENDIX: INTEGRATION OVER THE MATRICES $\mathcal{R}$.}

For the integration over the matrices $\mathcal{R}_{1}$ and $\mathcal{R}_{2}$ we make use of the fact that they can be parameterized as 34

$$
\mathcal{R}_{j}=-i \frac{\Delta}{2 \pi \hbar} U \hat{\tau}^{1 / 2} H_{j} \hat{\tau}^{1 / 2} U^{\dagger} \otimes \mathbb{1}_{2}, \quad j=1,2,
$$

where $U$ is an $N \times N$ unitary matrix, $\hat{\tau}$ is a diagonal $N \times N$ matrix containing the eigenvalues $\tau_{m}, m=1, \ldots, N$, of the Wigner-Smith time-delay matrix on the diagonal [38], $H_{j}$ is an $N \times N$ hermitian (real symmetric) matrix is time-reversal symmetry is broken (present), and $\mathbb{1}_{2}$ is the $2 \times 2$ unit matrix in spin grading.

For a chaotic quantum dot, the distributions of the hermitian matrices $H_{1}$ and $H_{2}$, the unitary matrix $U$, and the diagonal matrix $\hat{\tau}$ are all independent. The matrices $H_{j}, j=1,2$, have a Gaussian distribution,

$$
P(H) \propto \exp \left(-\beta \operatorname{Tr} H^{2} / 8\right),
$$

where $\beta=1$ if time-reversal symmetry is present and $\beta=2$ if time-reversal symmetry is broken by a magnetic field. The matrix $U$ is uniformly distributed in the unitary group, and the eigenvalues $\tau_{m}, m=1, \ldots, N$ of the time-delay matrix have distribution

$$
\begin{aligned}
P \propto & \left(1+\frac{e^{2}}{\pi \hbar C} \sum_{m=1}^{N} \tau_{m}\right) \prod_{m<n}^{N}\left|\tau_{m}-\tau_{n}\right|^{\beta} \\
& \times \prod_{m=1}^{N} \Theta\left(\tau_{m}\right) \tau_{m}^{-3 \beta N / 2+\beta-2} e^{-\beta \pi \hbar / \Delta \tau_{m}} .
\end{aligned}
$$

Knowing these distributions, finding the distribution for small $N$ becomes a matter of mere quadrature. We have obtained the plots of the distributions of $S^{P}$ and
$I / S^{P}$ by numerically generating $10^{7}-10^{8}$ matrices $\mathcal{R}_{j}$ distributed according to the above distribution. We refer to Ref. 35] for the details of implementation of this procedure. Moments of the noise and the current can be found by performing the Gaussian integrations over $H$ and the integrations over the unitary group with the help of the technique of Ref. [36]. For small $N$, the remaining integration over the $\tau_{m}$ can be done explicitly. For large $N$, it is sufficient to know the density and two-point correlator of the $\tau_{m}$ in order to find the first two moments of $I$ or $S^{P}$. The density of time-delays is 34

$$
\begin{aligned}
\rho(\tau) & =\sum_{m=1}^{N}\left\langle\delta\left(\tau_{m}-\tau\right)\right\rangle=\frac{N}{2 \pi \tau^{2}} \sqrt{\left(\tau_{+}-\tau\right)\left(\tau-\tau_{-}\right)} \\
\tau_{ \pm} & =2 \pi \hbar(3 \pm \sqrt{8}) / N \Delta
\end{aligned}
$$

The pair correlation function $K_{2}\left(\tau_{1}, \tau_{2}\right)$ is a universal function of the arguments $\tau_{1}$ and $\tau_{2}$ and the "spectrum edges" $\tau_{-}$and $\tau_{+} 44,45$. With the help of the pair correlation function 455 we find that, up to corrections of order $1 / N^{4}$,

$$
\left\langle\left(\sum_{m=1}^{N} \tau_{m}\right)^{q}\right\rangle=\left(\frac{2 \pi \hbar}{\Delta}\right)^{q}\left(1+q(q-1) \frac{2}{\beta N^{2}}\right) .
$$

[1] D. Thouless, Phys. Rev. B 27, 6083 (1983).

[2] Q. Niu, Phys. Rev. Lett. 64, 1812 (1990)

[3] L. P. Kouwenhoven, A. T. Johnson, N. C. van der Vaart, C. J. P. M. Harmans, and C. T. Foxon, Phys. Rev. Lett. 67, 1626 (1991).

[4] H. Pothier, P. Lafarge, C. Urbina, D. Esteve, and M. H. Devoret, Europhys. Lett. 17, 249 (1992).

[5] T. H. Oosterkamp, L. P. Kouwenhoven, A. E. A. Koolen, N. C. van der Vaart, and C. J. P. M. Harmans, Phys. Rev. Lett. 78, 1536 (1997).

[6] V. I. Fal'ko and D. E. Khmelnitskii, Zh. Eksp. Teor. Fiz. 95, 328 (1989) [Sov. Phys. JETP 68, 186 (1989)].

[7] B. Spivak, F. Zhou, and M. T. Beal Monod, Phys. Rev. B 51, 13226 (1995).

[8] P. W. Brouwer, Phys. Rev. B 58, 10135 (1998).

[9] F. Zhou, B. Spivak, and B. Altshuler, Phys. Rev. Lett. 82, 608 (1999).

[10] M. Switkes, C. M. Marcus, K. Campman, and A. C. Gossard, Science 283, 1905 (1999).

[11] T. A. Shutenko, I. L. Aleiner, and B. L. Altshuler Phys. Rev. B 61, 10366 (2000).

[12] J. E. Avron, A. Elgart, G.M. Graf, and L. Sadun, Phys. Rev. B 62, 10618 (2000).

[13] M. G. Vavilov, V. Ambegaokar, and I. L. Aleiner, Phys. Rev. B 63, 195313 (2001).

[14] M. Blaauboer and E. J. Heller, Phys. Rev. B 64, 241301 (2001). 
[15] A. V. Andreev and A. Kamenev, Phys. Rev. Lett. 85, 1294 (2000).

[16] L. S. Levitov, cond-mat/0103617 (2001)

[17] A. V. Andreev and E. G. Mishchenko, condmat/0104211.

[18] L. S. Levitov and G. B. Lesovik, Zh. Eksp. Teor. Fiz. 58, 225 (1993) [JETP Lett. 58, 230 (1993)].

[19] L. S. Levitov, H. Lee, and G. B. Lesovik, J. Math. Phys. 37, 4845 (1996).

[20] D. A. Ivanov and L. S. Levitov, Pis'ma Zh. Eksp. Teor. Fiz. 58, 450 (1993) [JETP Lett. 58, 461 (1993)]; D. A. Ivanov, H. Lee and L. S. Levitov, Phys. Rev. B 56, 6839 (1997).

[21] Yu. Makhlin and A. D. Mirlin, Phys. Rev. Lett. 87, 276803 (2001).

[22] M. Moskalets and M. Büttiker, cond-mat/0201259.

[23] M. Büttiker, Phys. Rev. B 46, 12485 (1992); Phys. Rev. Lett, 65, 2901 (1990); Phys. Rev. B 45, 3807 (1992).

[24] M. Büttiker, A. Prêtre, and H. Thomas, Phys. Rev. Lett. 70, 4114 (1993).

[25] M. Büttiker, J. Phys. Condens. Matter 5, 9361 (1993); M. Büttiker, H. Thomas, and A. Prêtre, Z. Phys. B 94, 133 (1994).

[26] M. Büttiker and T. Christen, in Quantum Transport in Semiconductor Submicron Structures, B. Kramer ed., NATO ASI Ser. E, Vol. 326 (Kluwer, Dordrecht, 1996).

[27] Y. M. Blanter and M. Büttiker, Phys. Rep. 336, 1 (2001).

[28] M. G. Vavilov, Ph.D. Thesis, Cornell University, (2001).

[29] M. G. Vavilov and I. L. Aleiner, Phys. Rev. B 60, R16311 (1999); 64, 085115 (2001).

[30] V. I. Yudson, E. Kanzieper, and V. E. Kravtsov, Phys.
Rev. B 64, 045310 (2001).

[31] M. Büttiker and R. Landauer, Phys. Rev. Lett. 49, 1739 (1982).

[32] The first order correction in an expansion in powers of $\hbar \omega / \gamma$ is given in Appendix C of Ref. 13. See also B. Wang, J. Wang, H. Guo, cond-mat/0107078 (2001), and O. Entin-Wohlman, A. Aharony, and Y. Levinson, condmat/0201073 (2002)

[33] P. W. Brouwer, S. A. van Langen, K. M. Frahm, M. Büttiker, and C. W. J. Beenakker, Phys. Rev. Lett. 79, 913 (1997).

[34] P. W. Brouwer, K. M. Frahm, and C. W. J. Beenakker, Phys. Rev. Lett. 78, 4737 (1997); Waves in Random Media 9, 91 (1999).

[35] J. N. H. J. Cremers and P. W. Brouwer, condmat/0110437 (2001)

[36] P. W. Brouwer and C. W. J. Beenakker, J. Math. Phys 37, 4904 (1996).

[37] M. L. Polianski and P. W. Brouwer, Phys. Rev. B. 64, 075304 (2001).

[38] E. P. Wigner, Phys. Rev. 98, 145 (1955), and F. T. Smith, Phys. Rev. 118, 349 (1960).

[39] C. W. J. Beenakker, Rev. Mod. Phys. 69, 731 (1997).

[40] M. L. Polianski and P. W. Brouwer, unpublished.

[41] J.E. Avron, A. Elgart, G.M. Graf, L. Sadun, Phys. Rev. Lett. 87, 236601 (2001).

[42] A. Alekseev, cond-mat/0201474.

[43] Y. Levinson, O. Entin-Wohlman, and P. Wölfle, condmat/0002187.

[44] E. Brézin and A. Zee, Nucl. Phys. B 402, 613 (1993).

[45] C. W. J. Beenakker, Nucl. Phys. B 422, 515 (1994). 